Korrekturbedarf bei Weiterbildungsordnung

\title{
Kaum Zeit für Kinder und Karriere?
}

Viele Ärztinnen stehen in der Weiterbildung vor hohen Hürden: Wegen der Familiengründung arbeiten Frauen häufig in Teilzeit - und sehen sich bei der Karriere ausgebremst. Es besteht Korrekturbedarf.

Die Weiterbildungsordnungen der Landesärztekammern und Familienfreundlichkeit stehen unter anderem nach Ansicht der Deutschen Gesellschaft für Hämatologie und Onkologie (DGHO) in einem klaren Missverhältnis. „Es gibt einen deutlichen Karriereknick für Ärztinnen während der Weiterbildung", erklärte Diana Lüftner, Berlin, auf einer Pressekonferenz anlässlich des Internationalen Frauentags. Ursächlich für dieses Dilemma sei die Anerkennung von Zeiten für die ärztliche Weiterbildung, die erst ab einem Stellenanteil von 0,5 möglich ist. „Das ist nicht mehr zeitgemäß. Eine Anerkennung ab einem Anteil von $25 \%$ wäre perspektivisch förderlich “, erklärt Lüftner. Diverse Fachgesellschafen fordert daher gemeinsam, die Bundesärz- tekammer sowie die diversen Landesärztekammern auf, die Weiterbildungsordnungen zu ändern.

\section{Gesellschaften fordern Anpassung}

Da eine Weiterbildung auf einer 0,5- oder 0,25-Stelle zwölf oder sogar 24 Jahre dauern würde, fordern die Fachgesellschaften hier eine Anpassung: Bei Erfüllung aller Inhalte und Bestätigung der notwendigen Fähigkeiten müsse die Weiterbildungszeit auf Basis einer Teilzeitbeschäftigung um bis zu $30 \%$ verringert werden können. Für viele junge Ärztinnen fällt in die Zeit der Weiterbildung auch die Familiengründung. Dabei erleben sie formale und organisatorische Hürden: So solle die Weiterbildung "grundsätzlich ganztägig“ absolviert werden. Weiterbildung in Teilzeit kann nur angerechnet werden, wenn die zuständige Kammer dies erlaubt. „Das birgt Unsicherheiten für Ärztinnen und zeigt, dass Weiterbildung in Teilzeit formal nicht akzeptiert wird“, sagt Maren Knödler vom Universitären Krebszentrum Leipzig. Sie hat gerade ihre Elternzeit beendet.
„Wenn wir eine moderne und im internationalen Vergleich konkurrenzfähige medizinische Forschung und Patientenversorgung wollen, dann brauchen wir moderne Arbeitsmodelle, die sich den gewandelten Familienmodellen anpassen", erklärt Anne Lesch, Berlin. Laut einer DGHO-Umfrage unter 2600 Mitgliedern glauben $71 \%$ der Teilnehmer - Frauen wie Männer -, dass die Vereinbarkeit von Familie und Beruf nur mit Kompromissen zu schaffen sei. 16\% der Frauen halten beides für unvereinbar. Und für einen großen Teil der weiblichen DGHO-Mitglieder heißt der Kompromiss: Kinderlosigkeit.

„Für viele Frauen ist eine Führungsposition der Verzicht auf Nachwuchs nicht wert", sagt Antonia Busse, Berlin. Um diese Frauen mit Kinderwunsch in den Kliniken, in der Patientenversorgung zu halten und auch für eine Führungsposition zu gewinnen, müssen neue, flexible Arbeitsmodelle geschaffen werden, lautet dei Forderung.

(Rebecca Beerheide)

Orthopädie \& Rheuma 2015/5

\section{Oskar Medizinpreis 2015 zum Thema Darmflora verliehen}

Die Berliner Stiftung Oskar-HeleneHeim vergibt den mit $50.000 €$ dotierten Oskar Medizinpreis | Forschungsarbeiten erbringen Erkenntnisse zum Stellenwert der Darmbakterien für die Gesundheit und Entstehung von Krankheiten

Der Oskar Medizinpreis 2015 wird geteilt und an zwei hochrangige Wissenschaftlerinnen verliehen. Professor Dr. Julia-Stefanie Frick, Universitätsklinikum Tübingen, und Privatdozentin Dr. Anja Sandek, Universitätsmedizin Göttingen, werden für wegweisende Forschungen zum Thema "Auswirkungen des Mikrobioms im Gastrointestinaltrakt" geehrt.

Der Darm ist das größte Organ des Menschen. In den letzten Jahren wurde die Darmflora (Mikrobiom) für die Wissenschaft immer wichtiger. Rapide gestiegene Erkenntnisse im Bereich der Mikrobiomforschung haben Hinweise auf metaboli- sche, immunologische, infektiologische neurologische und epidemiologische Einflussfaktoren des Mikrobioms auf die Pathophysiologie verschiedener Erkrankungen ergeben. Bereits Hippokrates und Luther waren überzeugt, dass der Darm einen gewichtigen Einfluss auf die Gesundheit hat.

Mit der Verleihung würdigt die Stiftung die wissenschaftliche Qualität der hochrangig publizierten Forschungsarbeiten dieser beiden Medizinerinnen über die Rolle der Darmflora (Mikrobiom) bei der Entstehung verschiedener Erkrankungen.

Die Forschungen von Professor Dr. Frick befassen sich damit, wie kommensale Bakterien mit dem Wirt und untereinander interagieren und neben lokalen entzündlichen und infektiösen Wirtsantworten auch systemische Immunantworten beeinflussen.

Privatdozentin Dr. Sandek hat ihre Forschung auf die Bedeutung des Gastrointestinaltrakts und des Mikrobioms für die Entstehung von systemischen Entzündungen bei Patienten mit chronischer Herzinsuffizienz ausgerichtet.

Das Preisgeld steht beiden Medizinerinnen für die Fortsetzung ihrer Forschungen zur Verfügung

Mitglieder der Jury für den Oskar Medizinpreis 2015 waren: Prof. Dr. Hans-Dieter Allescher (Klinikum Garmisch-Partenkirchen), Prof. Dr. Peter Bauerfeind (Universitätsspital Zürich), Prof. Dr. Thomas Frieling (HELIOS Klinikum Krefeld), Prof. Dr. Christoph Högenauer (Medizinische Universität Graz), Prof. Dr. Stefan MüllerLissner (Parkklinik Weißensee Berlin).

Der Oskar Medizinpreis 2015 wurde im Rahmen einer Vortragsveranstaltung der Stiftung Oskar-Helene-Heim zur Darmgesundheit am 26. Oktober 2015 in der URANIA Berlin verliehen.

Stiftung Oskar-Helene Heim, www.stiftung-ohh.de, Geschäftsführer: Werner Ukas,

Kontakt:werner.ukas@stiftung-ohh.de 